\title{
A Dynamical Model of Color Confinement*
}

\author{
S. Loh, T.S. Biró, U. Mosel and M.H. Thoma \\ Institut für Theoretische Physik, Universität Giessen \\ D-35392 Giessen, Germany
}

July 15, 2018

\begin{abstract}
A dynamical model of confinement based on a transport theoretical description of the Friedberg-Lee model is extended to explicit color degrees of freedom. The string tension is reproduced by an adiabatic string formation from the nucleon ground state. Color isovector oscillation modes of a $q \bar{q}$-system are investigated for a wide range of relative $q \bar{q}$-momenta and the dynamical impact of color confinement on the quark motion is shown.
\end{abstract}

*Work supported by BMBF and GSI Darmstadt. 
Kinetic theories are a well established tool in describing intermediate energy heavy-ion collisions. Their success has stimulated the development of transport theories based on QCD to describe collisions for even higher energies. For relativistic energies, string and parton cascade models (Fritjof [1], Venus [2], RQMD [3], HIJING [4], parton cascade [5]) have been developed. A serious problem of these models is the description of the final state of the reaction, the hadronization, which can no longer be treated perturbatively, since the hadrons are formed by the purely nonperturbative effects of color confinement. Therefore the dynamics of confinement should be considered in the transport descriptions.

There exist different approaches to deriving a transport theory that is based on an effective quark model incorporating the dynamics of the nonperturbative QCD vacuum. Zhang and Wilets [6] have derived a transport theory based on the Nambu JonaLasinio model in order to estimate chiral symmetry effects in heavy-ion collisions; this is conceptually close to our work. However, besides the absence of confinement, these authors do not actually perform dynamical simulations. Also soliton-soliton collisions in the framework of the Skyrme-model [7], [8] and of a Skyrme-like $\sigma$-model [9] have been performed. However, these calculations do not include any explicit quark degrees of freedom.

We assume that the nonperturbative effects can be modeled by the exchange of a scalar field, as it is successfully done in the Friedberg-Lee model (for a review see [10]). Since in this model the hadron surface is generated dynamically, it is well suited for dynamical simulations.

In recent articles [11, 12] we presented the derivation of the transport theory based on the Friedberg-Lee model and applied it to simulations of nucleon-nucleon collisions for bombarding energies of the order of a few $\mathrm{GeV}$. In this work color degrees of freedom were neglected. Here we present the derivation and first dynamical applications of a transport model with color confinement. After having fixed the strong coupling constant with the help of the string constant, we will investigate an excited state of the nucleon, the color isovector oscillation mode, showing the dynamical impact of 
color confinement on the quark motion.

The phenomenological Lagrangian of the Friedberg-Lee model reads [13, 14]

$$
\mathcal{L}=\bar{\Psi}\left(i \gamma_{\mu} \partial^{\mu}-m_{0}-g_{0} \sigma\right) \Psi+\frac{1}{2}\left(\partial_{\mu} \sigma\right)^{2}-U(\sigma)-\frac{1}{4} \kappa(\sigma) F_{\mu \nu}^{a} F_{a}^{\mu \nu}-i g_{v} \bar{\Psi} \gamma_{\mu} \frac{\lambda^{a}}{2} \Psi A_{a}^{\mu}
$$

where $\Psi$ denotes the quark fields, $\sigma$ is the color singlet scalar field representing the long range and nonabelian effects (of multi gluon exchange), and the last term contains the interaction of the residual abelian (one gluon exchange) color fields $A_{\mu}^{a}$

$$
F_{\mu \nu}^{a}=\partial_{\mu} A_{\nu}^{a}-\partial_{\nu} A_{\mu}^{a}
$$

All the nonabelian effects are assumed to be absorbed in the color dielectric function $\kappa(\sigma)$ which is chosen such that $\kappa$ vanishes as $\sigma$ approaches its vacuum value $\sigma=\sigma_{v}$ outside the bag and $\kappa=1$ inside. $U(\sigma)$ is the self-interaction potential for the scalar $\sigma$-field containing cubic and quartic terms. The Euler-Lagrange equations derived from (11) are

$$
\begin{aligned}
\left(\gamma^{\mu}\left(i \partial_{\mu}-i g_{v} \frac{\lambda_{a}}{2} A_{\mu}^{a}\right)-m_{0}-g_{0} \sigma\right) \Psi & =0 \\
\partial_{\mu} \partial^{\mu} \sigma+U^{\prime}(\sigma)+\frac{1}{4} \kappa^{\prime}(\sigma) F_{\mu \nu}^{a} F_{a}^{\mu \nu}+g_{0} \bar{\Psi} \Psi & =0 \\
\partial^{\mu}\left(\kappa F_{\mu \nu}^{a}\right) & =j_{\nu}^{a},
\end{aligned}
$$

where (3) is the Dirac equation describing the motion of the quarks, (44) is the KleinGordon equation for the time evolution of the $\sigma$-field, and (5) are the Maxwell equations for the gluon fields with the color charge currents $j_{\nu}^{a}=-i g_{v} \bar{\Psi} \gamma_{\nu} \frac{\lambda_{a}}{2} \Psi$. Solutions of these coupled set of nonlinear equations have up to now only been obtained in static calculations [13, 15, 16], since dynamical simulations suffer from instabilities, due to the small current quark mass and large Dirac-sea contributions. Here we will determine the dynamics of the quarks by a transport equation derived by Elze and Heinz [17]. These authors have shown on a semiclassical basis, that the following set of equations for the phase space distribution functions in the abelian limit can be derived:

$$
\left(p_{\mu} \partial^{\mu}-m^{*} \partial_{\mu} m^{*} \partial_{p}^{\mu}\right) f^{0}(x, p)=g_{v} p_{\mu} F_{a}^{\mu \nu} \partial_{\nu}^{p} f^{a}(x, p)
$$




$$
\left(p_{\mu} \partial^{\mu}-m^{*} \partial_{\mu} m^{*} \partial_{p}^{\mu}\right) f^{a}(x, p)=g_{v} p_{\mu} F_{a}^{\mu \nu} \partial_{\nu}^{p} f^{0}(x, p)
$$

Note that these equations (6) and (7) are the transport equations of motion from the Friedberg-Lee Lagrangian, whereas the corresponding equations (2.14) of [17] are derived from the QCD Lagrangian. $f^{0}$ and $f^{a}$

$$
f^{0}(x, p)=\int d Q f(x, p, Q) \quad, \quad f^{a}(x, p)=\int d Q Q^{a} f(x, p, Q)
$$

are the color singlet and the color octet distribution functions of the general distribution function $f(x, p, Q)$ for colored particles, with the space-time vector $x$, the four-momentum $p$ and an eight component c-number color vector, $Q=Q^{a}$. From these distribution functions we obtain the scalar density $\rho_{s}=\bar{\Psi} \Psi$, and the color charge density $j_{0}^{a}$ needed for the equations of motion (幽) and (5) by

$$
\rho_{s}=\frac{\eta}{(2 \pi)^{3}} \int d^{3} p \frac{m^{*}}{\omega} f^{0}(x, p) \quad, \quad j_{0}^{a}=\frac{\eta}{(2 \pi)^{3}} \int d^{3} p f^{a}(x, p)
$$

with the effective mass $m^{*}=m_{0}+g_{0} \sigma$ and the factor $\eta=4$ from spin and flavor degeneracy 12.

Because the color fields in (2) and (5) are purely abelian the equations (5) decouple to eight identical Maxwell equations. Adopting the Coulomb gauge $\vec{\nabla}(\kappa \vec{A})=0$, these equations can be written as 18

$$
\vec{\nabla}\left(\kappa \vec{\nabla} A_{0}\right)=-j_{0}
$$

Following the general assumptions of bag models [19], we assume that the contributions from the current $\vec{j}^{a}$ is already absorbed in the current quark mass $m_{0}$. Hence we focus on the colorelectric field described by (10) only, especially in view of $q \bar{q}$ production from strong colorelectric fields.

We make the following particular ansatz for $f^{0}$ and $f^{a}$

$$
\begin{gathered}
f^{0}(x, p)=f(x, p)+\bar{f}(x, p), \\
f^{1}(x, p)=\ldots=f^{7}(x, p)=0, \\
f^{8}(x, p)=f(x, p)-\bar{f}(x, p) .
\end{gathered}
$$


This ansatz corresponds to working in a U(1) subspace of SU(3)-color. This can be seen by remembering that there always exists an (abelian) gauge transformation such that the color vectors of the quarks contribute only to the commutating generators of $\mathrm{SU}(3), \hat{T}^{3}$ and $\hat{T}^{8}[20]$. Adopting this expansion, the quark $(f)$ and anti-quark $(\bar{f})$ distribution functions appearing in (11) and (13) can be interpreted as quark and $d i$-quark distribution functions. Nevertheless, we will in the following refer to $(\bar{f})$ as the anti-quark distribution function, the $\mathrm{U}(1)$ equivalent of the $\mathrm{SU}(3)$ di-quark. Inserting the ansatz (11)-(13) into the equations for the color moments (6) and (7) we end up with

$$
\begin{gathered}
\left(p_{\mu} \partial^{\mu}-m^{*}\left(\partial_{\mu} m^{*}\right) \partial_{p}^{\mu}\right) f(x, p)=g_{v} p_{\mu} F^{\mu \nu} \partial_{\nu}^{p} f(x, p) \\
\left(p_{\mu} \partial^{\mu}-m^{*}\left(\partial_{\mu} m^{*}\right) \partial_{p}^{\mu}\right) \bar{f}(x, p)=-g_{v} p_{\mu} F^{\mu \nu} \partial_{\nu}^{p} \bar{f}(x, p)
\end{gathered}
$$

which is a set of usual Vlasov equations describing the motion of charged particles in a selfconsistently generated scalar and vector field, determined by equation (1) and (10), respectively. The coupling of quark and antiquark degrees of freedom is therefore only provided by their interaction with the mean fields $\sigma$ and the colorelectric field.

Let us first examine the static limit of the transport equations. From quantum mechanics we know that the color charge density in a hadron has to vanish locally 21

$$
\int d^{3} p(f(x, p)-\bar{f}(x, p))=j_{0}^{8}(x)=<N\left|\hat{Q}^{8}(x)\right| N>=0,
$$

because the nucleon ground state $\mid N>$ is a color singlet and $\hat{Q}^{8}$ a color octet operator. From that we conclude that $f=\bar{f}$ and hence there is no colorelectric field in the groundstate. As shown by Vetter et. al [12], the distribution functions have to be of a local Thomas Fermi type

$$
f(x, p)=\bar{f}(x, p)=\Theta(\mu-\omega)
$$

with the Fermi energy $\mu$. After inserting the local Thomas Fermi distributions (17) into the integral expressions (8) the fermions can be integrated out, so that we are 
left with determining the soliton solution of the remaining $\sigma$-field equation (4). This solution is then obtained by standard methods used by Vetter et. al [12]. Comparing this solution with grounstate properties of the nucleon, the parameters of the model, $g_{0}$ and the ones of the potential $U(\sigma)$, can be fixed.

Since the colorelectric field vanishes in the static (ground state) solution (16), the strong coupling constant $\alpha_{s}=g_{v}^{2} / 4 \pi$ cannot be fixed from ground state properties. In order to determine its value we investigate the colorelectric energy in the cavity by forming a string like configuration. The string tension $\tau$, i. e. the coefficient of the linear rising $q \bar{q}$-potential

$$
V_{q \bar{q}}(r)=-\frac{k}{r}+\tau r \quad, \quad k=\frac{4 \alpha_{s}}{3}
$$

within soliton bag models has already been determined by several groups [15, 19, 22]. In these studies, the quark motion is neglected and an instantaneous electric field between them is assumed ab initio. We proceed differently and calculate the response of the colorelectric field to an adiabatic separation of the quark and anti-quark distributions with a constant velocity. Since we work in the testparticle picture [23], a fixed initial velocity $v_{z}$ is assigned to each particle belonging to $f$ and $-v_{z}$ to each particle belonging to $\bar{f}$. The velocity of the quarks is kept fixed during the time evolution. In this way the source densities for the time evolution of the $\sigma$-field and the instantaneous $A^{0}$-field equations (4) and (10) are obtained. Several values of $v_{z}$, ranging from $v_{z}=0.1 c$ to $v_{z}=0.5 c$, have been applied to assure the independence of the colorelectric field on $v_{z}$.

At the beginning of the motion we see in fig. 1 how the cavity is deformed by the motion of the quarks $(t=3 \mathrm{fm})$. While the charge distributions move apart the colorelectric field, as a solution of (10), builds up (fig. 2]a). The genuine feature of this field is, that it is parallel everywhere at the boundary of the cavity, which is due to the von Neumann boundary conditions, forcing the normal component of the displacement field $\vec{D}=\kappa \vec{E}$ to vanish. Hence we find the typical string like behaviour of the $\vec{E}$-field in the later stages of the time evolution (fig. 2 $b$ ), namely being 
constant and almost independent of the axial coordinate in the region between the quarks. Determining the colorelectric energy $E_{\text {glue }}=\frac{1}{2} \int d^{3} r \vec{D} \vec{E}$ as a function of the $q \bar{q}$-distance provides us with a measure for the string constant $\tau$. Depending on the value of $\alpha_{s}$ the string constant is shown in fig. . In order to obtain the generally accepted value of $\tau \approx 1 \mathrm{GeV} / \mathrm{fm}$, we need to have values of $\alpha_{s} \approx 2$, which is in agreement with the values of MIT-bag and other Friedberg-Lee model calculations [15, 19, 22].

Having fixed the strong coupling constant, we now perform the time evolution of the full model equations for the $\sigma$-field (4), the gluon field (10) and the transport equations (14) - (15). As a first example, we excite the soliton ground state at time $t=0$ by shifting the equilibrium (Thomas Fermi) momentum distribution (17) of the quarks with a fixed momentum $+p_{z}$ and that of the anti-quarks with $-p_{z}$. (Values used for $p_{z}$ range from $p_{z}=\mu=350 \mathrm{MeV}$ to $p_{z}=30 \mu$ ). A self-consistent time evolution of the equations of motion (41) and (14)-(15) is then generated by a numerical integration sceme as used by Vetter [12]. The instantaneous colorelectric field is determined in every timestep of the evolution by a finite element method developed by Mitchell [24]. The general features of the resulting process can be summarized as follows:

Depending on their initial momenta, the quarks move apart along the z-axis and the electric field builds up. As a consequence, the quarks lose their kinetic energy to the $\vec{E}$-field and additionally to the deformation of the cavity to a string like configuration. When the entire kinetic energy is deposited into the $\vec{E}$ - and $\sigma$-field, the motion is stopped and starts to reverse. The quarks then pass through each other at the origin and the process is repeated in the opposite direction, leading to an oscillation of the $q \bar{q}$-system. If we follow the process further, the coherent motion of the $q \bar{q}$-system is disturbed due to an increasing chaotic motion of the testparticles inside the deformed cavity [25]. As a consequence, the strength of the colorelectric field decreases as shown in (fig. (4) for an initial energy of $3 \mathrm{GeV}$, accompanied by a steady elongation of the cavity. This behaviour continues until the total loss of the 
colorelectric energy is used for a large amplitude vibration of the bag (fig. 5). For very large times the superposition of the $q$ and $\bar{q}$ distributions is complete and the colorelectric field vanishes, so that the final state is again a locally color neutral one as it was at $t=0$.

Note that the elongation of the cavity shown in fig. 5 is caused by the initial momentum $p_{z}$ that acts as an additional pressure on the cavity. A further effect of the chaotic motion to be expected is the damping of multipole modes in Vlasov-type transport models [25], leading to an equal distribution of the particle momenta to all degrees of freedom. This damping can hardly be seen in our particular scenarios, since the excitation energies (typically of the order of a few $\mathrm{GeV}$ ) are too large, so that the equipartition of the particle momenta happens on a much larger time scale. For lower exitation energies of $\approx 300 \mathrm{MeV}$, however, this one body friction effect, driving the bag to a spherical shape, can be seen.

Summarizing the statements above, we can say that independent of the relative momentum of the quarks, the motion is always restricted to the cavity by the mutual interaction of the $\vec{E}$-field and the $\sigma$-field with the quarks inside the dynamically deformed cavity. Thus, the inherent feature of the Friedberg-Lee model, color confinement, is maintained in our dynamical model, i. e. for an arbitrary momentum of the quarks.

Further applications of our model will be the effects of color gluon fields in nucleonnucleon collisions, similar to our investigations in the colorless model [11, 12], or the description of quark gluon plasma scenarios, in particular the dynamical treatment of the hadronization process. Also a consistent description of $q \bar{q}$-production can be implemented via the Schwinger mechanism. Work along this direction is in progress. 


\section{References}

[1] B. Andersson, G. Gustafson, B. Nielsson-Almquist, Nucl. Phys. B281 (1987) 289

[2] K. Werner, Z. Phys. C42 (1989) 85

[3] H. Sorge, H. Stcker and W. Greiner, Nucl. Phys. A498 (1989) 567c

[4] X.-N. Wang and M. Gyulassy, Phys. Rev. D44 (1991) 3501

[5] K. Geiger and B. Mller, Nucl. Phys. B369 (1992) 600

[6] W.-M. Zhang and L. Wilets, Phys. Rev. C45 (1992) 1900

[7] J. J. M. Verbarschoot, T. S. Walhout, J. Wambach and H. W. Wyld, Nucl. Phys. A461 (1987) 603

[8] A. E. Allder, S. E. Koonin, R. Seki, and H. M. Sommermann, Phys. Rev. Lett. 59 (1987) 2836

[9] A. Kudrayavtsev, B. Piette, and W. J. Zakrzewski, Z. Phys. C60 (1993) 731

[10] L. Wilets, Nontopological Solitons, Lecture Notes in Physics, Vol. 24 (World Scientific, Singapore 1989)

[11] U. Kalmbach, T. Vetter, T. S. Biró and U. Mosel, Nucl. Phys. A563 (1993) 584

[12] T. Vetter, T. S. Biró and U. Mosel, Nucl. Phys. A581 (1995) 598

[13] R. Friedberg and T.D. Lee, Phys. Rev. D15 (1977) 1694 and D16 (1977) 1096

[14] R. Friedberg and T.D. Lee, Phys. Rev. D18 (1978) 2623

[15] M. Bickebller, M.C. Birse, H. Marschall and L. Wilets, Phys. Rev. D31 (1985) 2892

[16] M. Bickebller, M.C. Birse and L. Wilets, Z. Phys. A326 (1988) 89 
[17] H.Th. Elze, U. Heinz, Phys. Rep. 183 (1989) 81

[18] M. Bickebller, R. Goldflam, and L. Wilets, J. Math. Phys. 26 (1985) 1810

[19] T. DeGrand, R. L. Jaffe, K. Johnson, J. Kiskis, Phys. Rev. D 12 (1975) 2060

[20] P. Sikivie, N. Weiss, Phys. Rev D 18 (1978) 3809

[21] U. Mosel, Fields, Symmetries and Quarks, McGraw-Hill (1989)

[22] M. Grabiak, M. Gyulassy, J. Phys. G 17 (1991) 583

[23] C. Y. Wong, Phys. Rev. C 25 (1982) 1460

[24] W. F. Mitchell, Unified Multilevel Adaptive Finite Element Methods for Elliptic Problems, PhD Thesis Unversity of Illinois, (1988)

[25] G. F. Burgio, M. Baldo, A. Rapisarda, Phys. Lett. B321 (1994) 307; Nucl. Phys. A583 (1995) 343; Phys. Rev. C51 (1995) 198 
Figure 1: Baryon density $\rho=\int(f+\bar{f}) d^{3} p$ (left) and the $\sigma$-field (right) at time $t=3 \mathrm{fm}$ (upper) and $t=40 \mathrm{fm}$ (lower). The equidistant lines of constant value start at $0.1 \mathrm{fm}^{-3}$ for the quark density $\rho$ with an increase of $0.1 \mathrm{fm}^{-3}$. The values for $\sigma$ start at $-0.125 \mathrm{fm}^{-1}$ with an increase of $0.05 \mathrm{fm}^{-1}$.

Figure 2: The electric field $\vec{E}$ inside the cavity. a) At time $t=3 \mathrm{fm} / \mathrm{c}$ and b) at time $t=40 \mathrm{fm} / \mathrm{c}$. Note, that b) has a rescaled z-axis to fit the page.

Figure 3: String constant $\tau$ as a function of the strong coupling constant $\alpha_{s}$.

Figure 4: Time evolution of the colorelectric energy up to $t=100 \mathrm{fm} / \mathrm{c}$ resulting from an exitation energy of $3 \mathrm{GeV}$ (dotted line). For the solid line the strong oscillations have been averaged out.

Figure 5: Expectation value of the $z$-coordinate $\left\langle z>=\iint z f^{0}(x, p) d^{3} x d^{3} p\right.$ as a function of the time $t$ resulting from an exitation energy of $3 \mathrm{GeV}$ (dotted line). For the solid line the oscillations have been averaged out. 

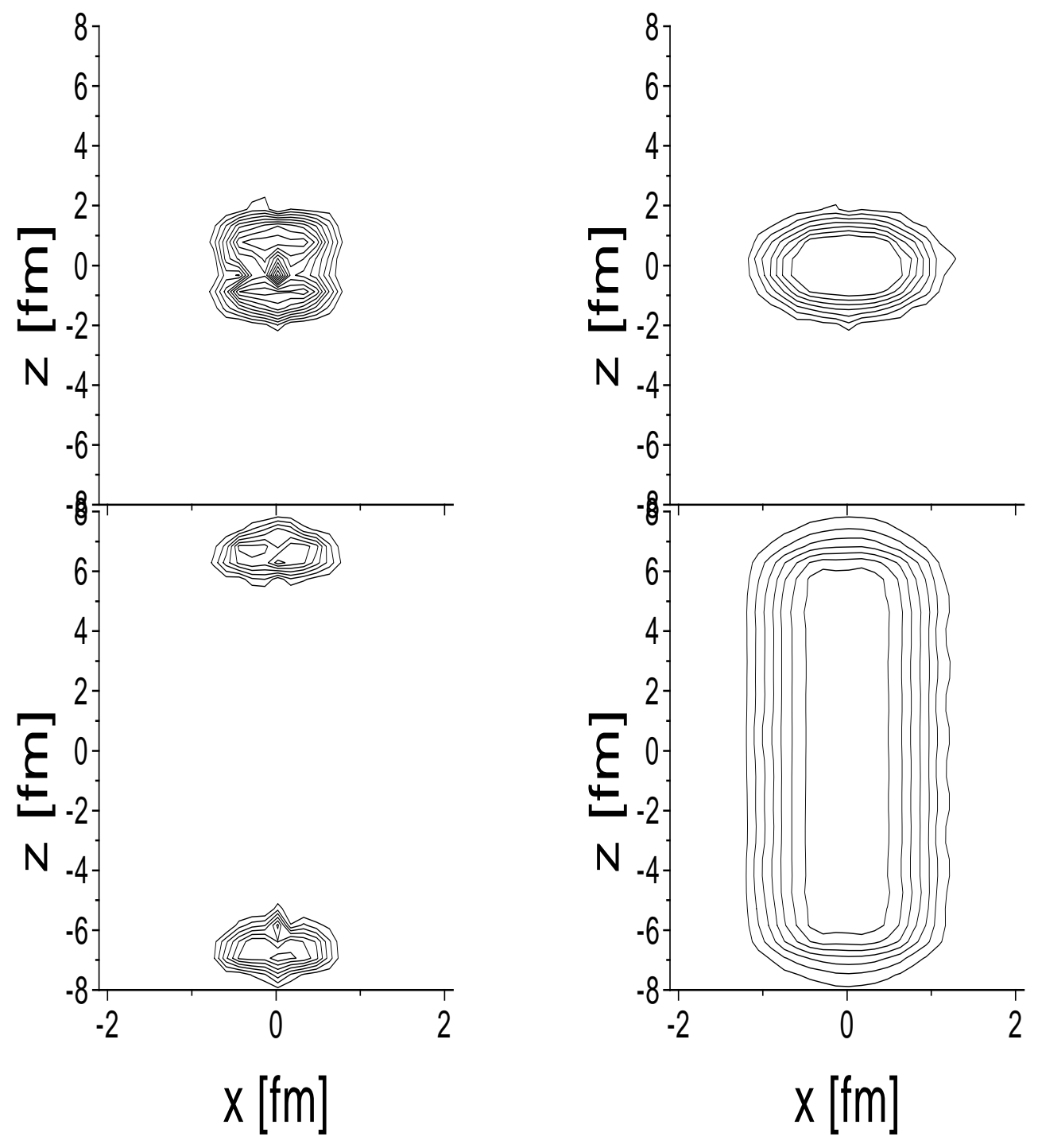

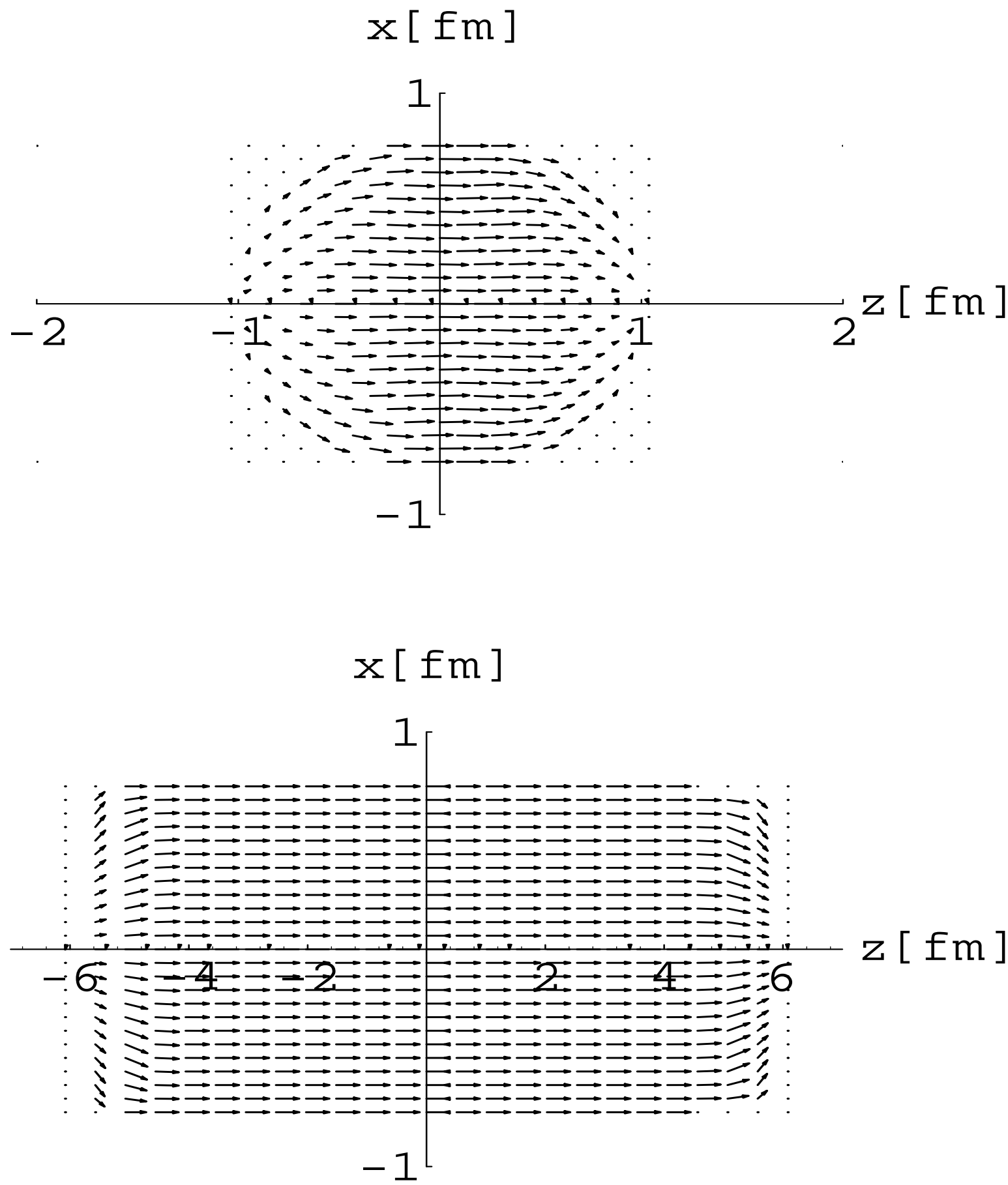


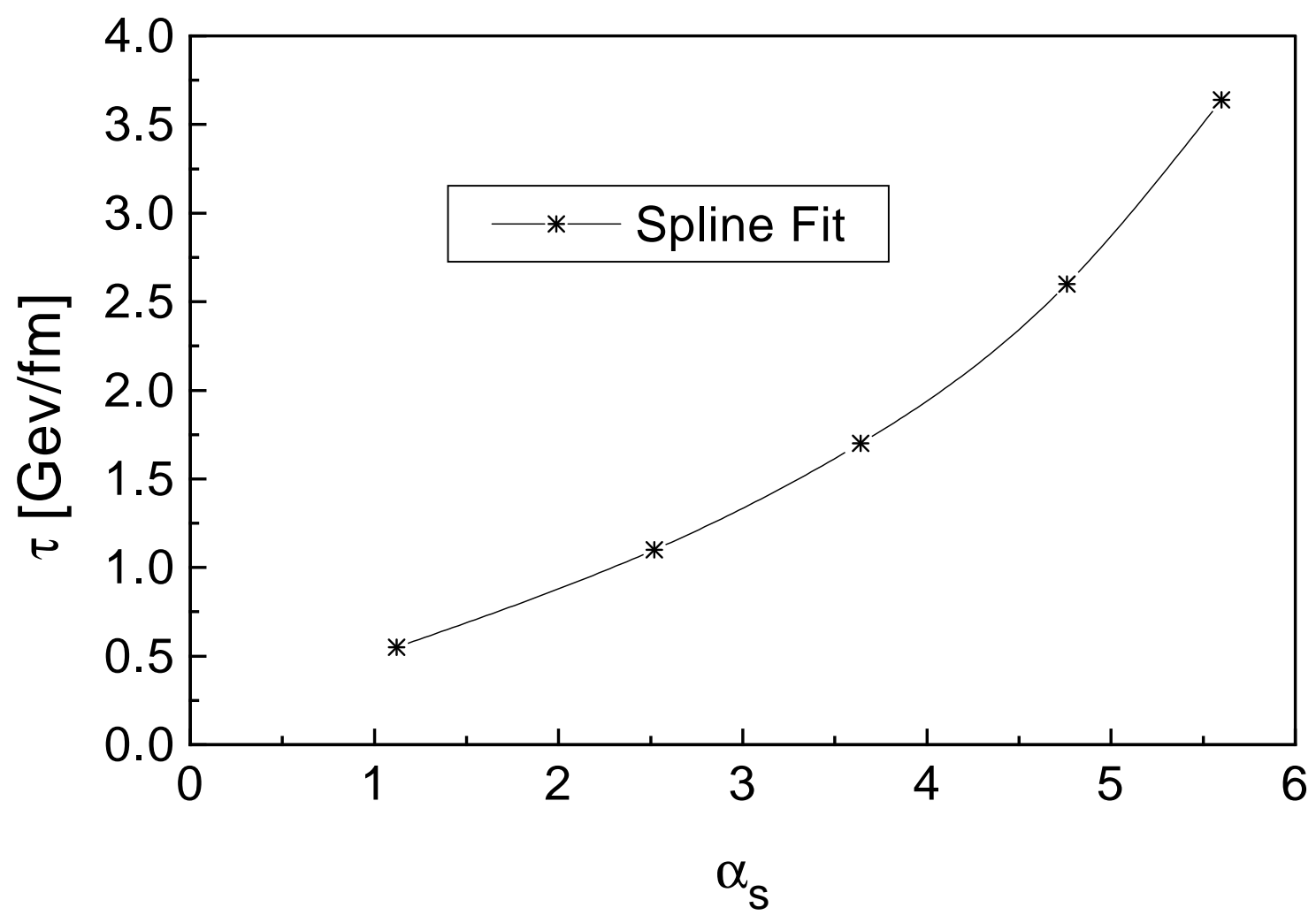




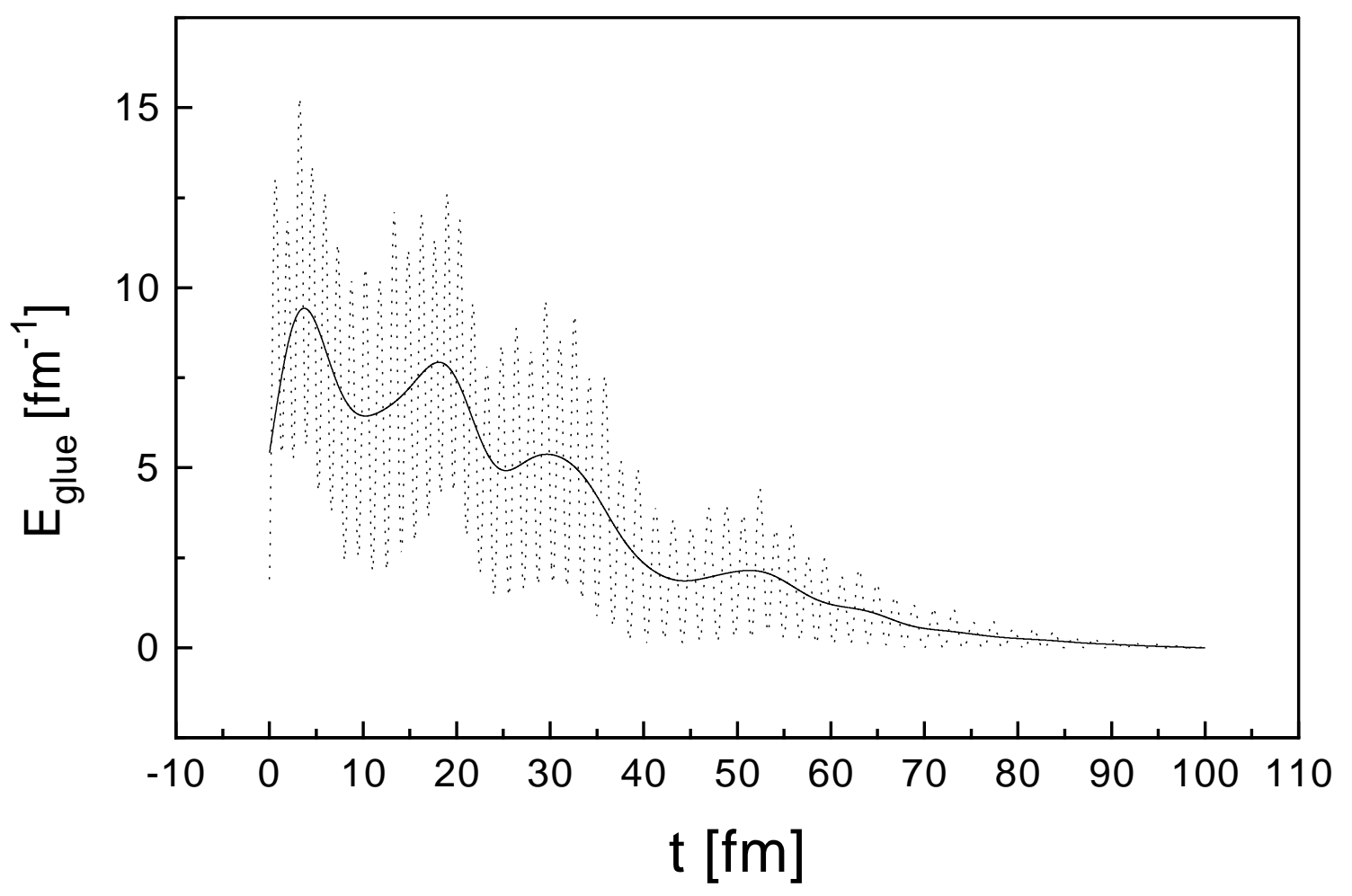




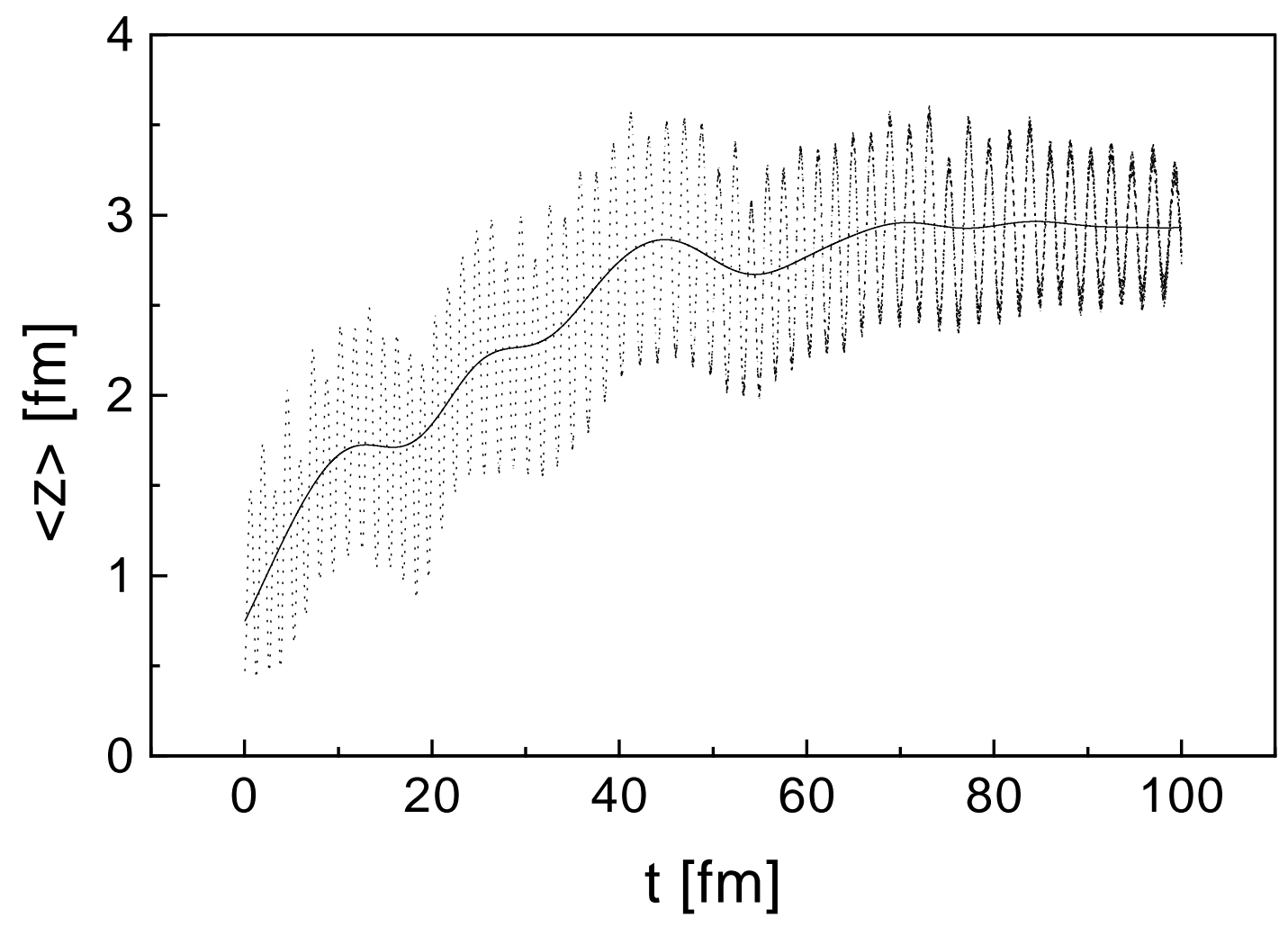

